\section{Modelo dinámico para proyectar la necesidad de recursos humanos en salud: anestesistas en Uruguay}

\author{
Anesthesiologists in Uruguay: Dynamic model to project \\ the need of human resources in healthcare
}

Fiorella Cavalleri, Ángel Segura, Marisa Buglioli, Juan Riva y Patricia Barber

Recibido 9 septiembre 2020 / Enviado para modificación 23 agosto 2021 / Aceptado 28 octubre 2021

\section{RESUMEN}

Objetivo Desarrollar un modelo dinámico para simular la oferta de médicos especialistas y estimar la brecha especto a la demanda/necesidad en anestesiología en el sistema de salud del Uruguay.

Métodos Se desarrolló un modelo de simulación dinámico determinístico implementado en el programa libre R. Se analizaron las proyecciones en el período 2011-2050 y se estimó la brecha a partir de la situación de equilibrio o desequilibrio entre oferta y demanda/necesidad. Se evaluó la calidad del modelo comparando los valores simulados con los datos históricos, con indicadores de bondad de ajuste, como la raíz del error cuadrático medio relativo (rRMSE).

Se realizó un análisis de sensibilidad con respecto a los cupos de ingreso a la especialidady la tasa de crecimiento de la necesidad de especialistas.

Resultados Se proyectó la oferta y demanda de anestesistas para el período considerado. Se obtuvo un rRMSE menor a 0,1 , lo que sugiere que el modelo propuesto reproduce adecuadamente la dinámica de la oferta real. Para el período proyectado la situación a mediano y largo plazo es de equilibrio.

Conclusión El modelo simulado presenta buen ajuste, por lo que la proyección de la oferta de Recursos Humanos (RR. HH.) representa de forma precisa la disponibilidad futura de la fuerza de trabajo. Además, el modelo representa un insumo de interés para la gestión informada sobre la necesidad de recursos humanos y las políticas de salud, dado que permite evaluar las proyecciones bajo diferentes escenarios.

Palabras Clave: Recursos humanos, planificación en salud, proyección (fuente: DeCS, BIREME).

\section{ABSTRACT}

Objectives The aim of this study is to develop a dynamic model to simulate the supply of specialized physicians in Anesthesiology and estimate the gap with its demand, within Uruguay healthcare system.

Methods A deterministic dynamic simulation model was developed and implemented using R software. Projections for the 2011-2050 period were analyzed, and the gap was estimated based on the equilibrium state of supply and demand. The quality of the model was evaluated comparing the simulated data with historical empirical data using goodness of fit indicators, such as the relative root mean square error (rRMSE).

Results The demand and supply of anesthesiologists was projected for the period under analysis. A rRMSE $<0,1$ was obtained, which suggests the proposed model adequately reproduces the real offer dynamics. Based on the defined gap criteria, in the medium and long-term the situation is in equilibrium state.

Conclusions The simulated model presents a good fit so that the human resources (HR) supply projection represents in a precise way the future availability of the workforce. Given that the model allows to evaluate the projection dynamics under different
Artículo / Investigación Article / Research
FC: Lic. Economía. Ph.D.(c) Ciencias Biomédicas. Departamento de Medicina Preventiva y Social. Facultad de Medicina, Universidad de la República. Montevideo, Uruguay.

fcavalleri1226@gmail.com

AS: Lic. Ciencias Biológicas. Ph.D. Ecología. Modelización y Análisis de Recursos Naturales, Centro Universitario Regional Este. Universidad de la República.

Rocha, Uruguay.

asegura@cure.edu.uy

MB: MD. M. Sc. Economía de la Salud.

Departamento de Medicina Preventiva y Social. Facultad de Medicina, Universidad de la República. Montevideo, Uruguay. marisa.buglioli@gmail.com

JR: MD. Esp. Anestesiología. Departamento de Anestesiología. Facultad de Medicina,

Universidad de la República.

Montevideo, Uruguay.

juanrivafortunato@gmail.com

PB: Lic. Economía. Ph.D. Economía.

Universidad de Las Palmas de Gran Canaria, Campus Universitario de Tafira. Las Palmas de Gran Canaria, España.

patricia.barber@ulpgc.es 
management scenarios, the model also represents an input of the utmost interest for management knowledgeable about human resource demands and healthcare policy.

Key Words: Workforce, health planning, projection (source; MeSH, NLM).

$\mathrm{E}$ desequilibrio entre la oferta y la demanda de recursos humanos para la atención de la salud es un problema mundial (1). En particular, la escasez de médicos es un desafío común no solo en las naciones más desarrolladas, sino también en los países en desarrollo (1). Desde organismos internacionales se señala que, a nivel agregado, la disponibilidad de personal de salud está aumentando en la mayoría de los países de los que se dispone de datos, aunque no lo suficientemente rápido como para mantener el ritmo de crecimiento de las necesidades de la población. Los principales problemas identificados son: la escasez de profesionales, su mala distribución geográfica, las barreras en la colaboración profesional, el uso ineficiente de los recursos, las malas condiciones de trabajo y la disponibilidad limitada de datos sobre la fuerza de trabajo de salud (2).

En Uruguay, desde la autoridad sanitaria, se señala que el país se encuentra en una situación privilegiada en cuanto a disponibilidad de profesionales de la salud. En el caso del personal médico, se registran 47,7 médicos por 10 ooo habitantes (3). Desde el Ministerio de Salud, se argumenta que "Uno de los principales temas pendientes a abordad desde la política pública es la planificación en materia de recursos humanos en salud. Las complejidades del mercado de trabajo hacen imperioso avanzar en esta temática" (3, p. 66). Se destacan como principales problemas del mercado de trabajo médico: el multiempleo, con 2,25 empleos por médico en promedio, la heterogeneidad en las cargas horarias semanales, los problemas asociados a la distribución territorial o a los desequilibrios en composición de los recursos humanos sanitarios en general y de médicos en particular (3).

En la Ley 19301, referente al Sistema Nacional de Residencias Médicas (4), se establece que la distribución cuantitativa de los médicos residentes en los servicios de salud acreditados será realizada por el Consejo Administrador Honorario del Sistema Nacional de Residencias Médicas. En este sentido, es prioritario contar con metodologías rigurosas y datos de calidad para que los planificadores puedan tomar decisiones informadas.

Para proyectar la fuerza laboral médica se han desarrollado diversas metodologías. Los enfoques básicos se fundamentan en algunas de las siguientes dimensiones: demanda, necesidad, oferta y benchmarking $(1,5-6)$. Los modelos de planificación de la fuerza laboral pueden combinar uno o más de estos enfoques, en función de los objetivos planteados y, en cualquier caso, abordarse con diferentes aproximaciones metodológicas, cada una con sus fortalezas y limitaciones (5).
Particularmente, los modelos de simulación se han erigido en una herramienta potente para planificar las necesidades de recursos humanos (RR. HH.) mediante una metodología que permite visualizar el comportamiento de un sistema real a lo largo del tiempo. Entre sus cualidades se destaca que permiten responder variaciones en las condiciones o forzantes del sistema y conocer la evolución del sistema analizado ante diferentes hipótesis y escenarios de análisis.

Una de las técnicas de simulación para la proyección de la oferta y demanda de RR. HH. que están incorporando prestigiosos centros y observatorios de planificación de recursos humanos es la dinámica de sistemas (DS).

La Ds utiliza técnicas de integración numérica para estudiar sistemas complejos retroalimentados basados en una estructura de niveles y flujos que representan el sistema original (7).

La estructura de un sistema y las relaciones que existen entre sus variables produce patrones de comportamiento dinámico de las variables del sistema. El objetivo de los modelos dinámicos es comprender el comportamiento de un sistema y cómo es afectado por las variables que es posible modificar. Esta comprensión normalmente produce el marco para determinar qué acciones pueden mejorar el sistema o solucionar sus problemas.

En los últimos años, el modelado de sistemas dinámicos se ha desarrollado ampliamente como un método adecuado para proyectar los recursos humanos para la salud (1,6-12). Particularmente, Ishkawa et al. (10) estiman los déficits y superávits de médicos especialistas en Japón para especialidades clínicas con un modelo de Ds con un horizonte temporal en 2030. Recientemente, Senese et al (13) utilizaron la Ds para simular las necesidades de recursos humanos en el sector sanitario. Desarrollan un modelo de DS para proyectar la oferta de médicos especialistas en una región italiana bajo tres escenarios de demanda con horizonte temporal en 2030. Lo combinan con un modelo de programación lineal para garantizar la asignación adecuada de plazas de formación. Ansah et ál. (14) modelaron las necesidades de especialistas en oftalmología en Singapur construyendo un modelo para simular la prevalencia de enfermedades oftalmológicas, de demanda y de requerimientos de especialistas en el sector público del país.

El Observatorio de Recursos Humanos para la salud del Reino Unido, el Centre for Workforce Intelligence (CFWI) ha adoptado DS para sus modelos de planificación de recursos humanos para la salud, incluso para la 
especialidad de anestesiología (15-17). En una revisión del año 2016, el CfWI (18) realizó una reflexión histórica sobre la aplicación del Ds para modelar la fuerza laboral en la atención integral a la salud en Inglaterra. Al inicio se usaron planillas de cálculo y/o el programa $\mathrm{R}$ para desarrollar los modelos y se basaron en conceptos estándar de Ds, como niveles, flujos, retardos y retroalimentación. A partir de 2012, CfWI realizó la transición al uso de un programa específico (VENSIM) para simular modelos complejos con la misma metodología. Los programas informáticos utilizados para la modelización son un punto clave. Es deseable que las distintas comunidades generen formación y experiencia, pero, fundamentalmente, a partir del uso de programas libres, de código abierto, a fin de formar comunidades que contribuyan al desarrollo del conocimiento.

Masnick y MacDonnell (19) elaboraron un ambicioso modelo basado en Ds que integra las necesidades de recursos humanos relacionados con la salud de la población y con el sistema de organización sanitaria.

Barber et ál. (2010) llevarona cabo modelos de simulación de brecha de oferta demanda/necesidad para especialistas médicos en el sistema de salud de español y actualizaciones periódicas a partir de la metodología de Ds (20-24).

Como antecedente hemos desarrollado para Uruguay un modelo de proyección para evaluar brechas a nivel nacional. Además, se analizaron las proyecciones a futuro y se discutieron distintos escenarios para las especialidades de anestesiología y pediatría (5-6). Se hizo un estudio para estimar necesidad de anestesistas en 2011 , a partir de la estimación de horas necesarias de anestesistas (25). Sin embargo, en Uruguay no hay antecedentes de aplicación sistemática de modelos para analizar la evolución de la fuerza de trabajo ni de proporcionarles a los planificadores, tomadores de decisiones en salud, formadores de recursos humanos sanitarios, evidencia basada en proyecciones de demanda/necesidad y oferta para indicar la trayectoria del sistema bajo diferentes condiciones. Tampoco se dispone de antecedentes que aporten como "banco de pruebas" para evaluar el impacto de diferentes intervenciones (incremento de cupos para las residencias, tiempo de duración de la formación, variaciones en la edad jubilatoria, duración de la jornada laboral).

Los modelos deberían hacer uso de datos disponibles teniendo en cuenta que, históricamente, las fuentes de datos utilizadas en la planificación de RR.HH. se recogen para un propósito primario diferente; por ejemplo, el registro de profesionales, monitoreo y gestión de personal de salud. En consecuencia, requieren un tiempo considerable para acceder, interpretar, verificar y estandarizar.

En Uruguay las bases de datos sistematizados son relativamente recientes y presentan desafíos en cuanto a su calidad y completitud. A ello se suma que la "modalidad de trabajo médico" se caracteriza por el multiempleo, la variabilidad de la edad jubilatoria y la heterogeneidad en cantidad de horas trabajadas por semana dentro del mismo Sistema Nacional Integrado de Salud. La construcción de modelos explícitos nos permite identificar vacíos de información y variables clave que se deben monitorear.

Queda en evidencia que aún modelos con bajo nivel de complejidad estructural, con un número reducido de variables, implican un desafío en cuanto a la disponibilidad de información de calidad necesaria para desarrollarlos y evaluarlos.

Este estudio tiene como propósito generar un modelo que les sirva de insumo a los responsables de las políticas de RR. HH. en la salud. Su objetivo general es elaborar un modelo de simulación basado en la evaluación de los desequilibrios entre la oferta y demanda/necesidad de médicos especialistas en anestesiología en el Sistema de Salud del Uruguay (201 1-2050).

\section{MÉTODOS}

\section{Modelo de simulación}

Se ha diseñado un modelo de simulación dinámico basado en la metodología de Ds desarrollada por Forrester en 1958 (19). Se utilizó el programa R (R Core Team [2016]. R: A language and environment for statistical computing. R Foundation for Statistical Computing, Vienna, Austria. URL https://www.R-project.org/) para la implementación del modelo.

La primera etapa es crear los diagramas de bucles causales, a través de los cuales se identifican las estructuras e interacciones de los circuitos de retroalimentación que caracterizan a los modelos dinámicos. Consisten en diagramas y enlaces causales queconectan una variable de causa con una variable de efecto (19-23).

Los diagramas de nivel y flujo permiten analizar cuantitativamente el comportamiento de un sistema dinámico. En ellos se distinguen dos tipos de variables: Las variables de nivel o stock describen los estados del sistema; por ejemplo, el número de especialistas en un momento del tiempo. Por su parte, las variables de flujo muestran las tasas de cambio de los niveles; por ejemplo, la cantidad de puestos para ingresar a estudiar la especialidad. Las variables de nivel son acumulaciones de flujos y se calculan matemáticamente como:

$$
\begin{gathered}
\operatorname{Nivel}_{\text {especialistas }}(t)=\frac{\Delta(\text { especialistas })}{\Delta(t)}+\operatorname{Nivel}_{\text {especialistas }}(t-1) \\
\frac{\Delta(\text { especialistas })}{\Delta(t)}=\frac{\Delta(\text { especialistas })^{t}}{t-(t-1)}=\left(\text { flujos }_{\text {entrada }}\right)-\left(\text { flujos }_{\text {salida }}\right)
\end{gathered}
$$

Se diseñó un modelo teórico y las relaciones causales para luego correr la simulación de especialistas en anes- 
tesiología en Uruguay. De esta forma se representan los aspectos más relevantes del sistema. Se considera un horizonte temporal para la proyección hasta 2050, con el objetivo de analizar la dinámica de largo plazo.

La estructura del modelo tiene dos componentes: oferta y demanda/necesidad. Los años de inicio de los componentes corresponden a distintos años, dependiendo de la disponibilidad de información. Las estimaciones hasta 2017 fueron para poder calibrar y evaluar el modelo en base a datos reales. El período de proyección desde 2018 hasta 2050 deberá ser valorado en el futuro.

El modelo de oferta (Figura 1) muestra el ciclo de vida laboral de los especialistas desde la etapa de formación hasta la jubilación o muerte. Se consideró el ingreso a la residencia (que en el Uruguay es específica para cada especialidad) y, en cuanto a los ingresos, se cuenta con datos del 2000 al 2017, proporcionados por Escuela de
Graduados de la Universidad de la República. La formación en anestesiología dura 4 años. Respecto al total de estudiantes de la especialidad que cursan los distintos años (residentes de primer $a \tilde{n} o=R_{1}$, de segundo $\mathrm{año}=\mathrm{R}_{2}$, de tercero $=\mathrm{R}_{3}$ y de cuarto año $=\mathrm{R}_{4}$ ), se cuenta con información del 2010 a 2016 (Departamento de Anestesiología de la Universidad de la República). Para el período señalado se cuenta con datos del total de residentes, los nuevos ingresos a estudiar la especialidad, los que habiendo culminado los cursos deben la prueba final para obtener el título de especialista y los que egresan con el título de especialista.

Sobre el total de profesionales se cuenta con datos histórico para los años 2000 a 2017.

Las tasas de mortalidad utilizadas fueron las de población general, tomadas del Instituto Nacional de Estadística (INE).

Figura 1. Diagrama causal y diagrama de niveles y flujos

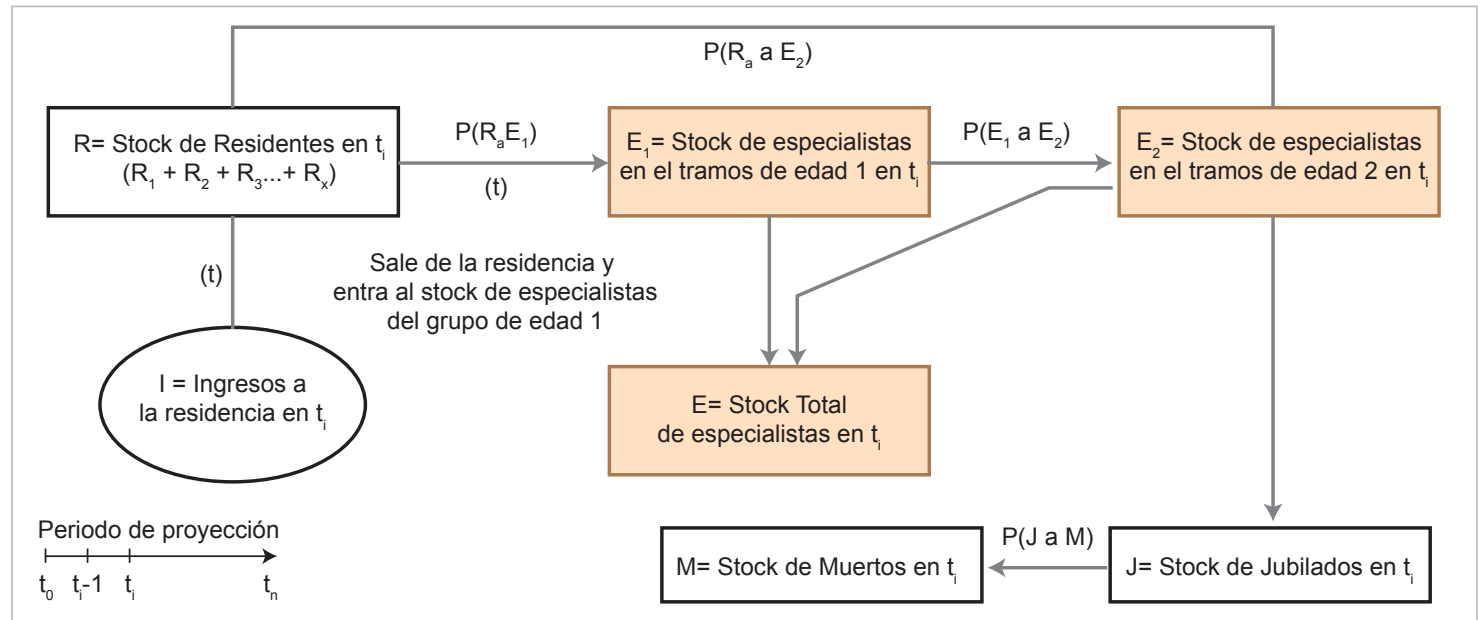

I = Cantidad de residentes que ingresan cada año a estudiar la especialidad según datos de Escuela de la Graduados. Desde el año 2000 al 2016 en promedio ingresaron 15 personasa la residencia con unvalor máximo de 30 ingresos; $R=$ Total de residentes en formación de todos los años (R1, R2, R3 y R4). Valor que en promedio ha sido de 90 residentes; $E_{1}=$ Cantidad de anestesistas activos jóvenes, que ingresan al mercado de trabajoen el primer tramo de edad considerado (entre 32 y 37 años aproximadamente); $\mathrm{E}_{2}=$ Número deanestesistas activos que ingresan al mercado de trabajo con edades en el segundo tramo de edad

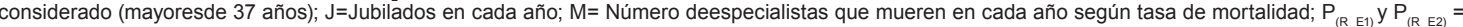
Probabilidades de pasar de residente a especialista en el tramo de edad 1 y 2 respectivamente; $P_{(E 1 E 2)}=$ Probabilidad de que el especialista pase del grupo de edad 1 algrupo de edad 2; $P_{(E 2 J)}=$ Probabilidad de que el especialista pase del grupo de edad 2 a jubilarse; $P_{(E 2 ~ J)}=$ Probabilidad de pasar de jubilado a muerto.

La probabilidad de transición de residente (R) a Especialista (E), se estimó a partir de frecuencias históricas de cantidad total de residentes y cantidad de egresados por año; en promedio, aproximadamente el $26 \%$. Este $26 \%$ se desglosa en un $19 \%$ de probabilidad de transitar de residente a especialista dentro del primer tramo de edad, y en un $7 \%$ de probabilidad de transitar de residente a especialista, pero en edades más tardías.

Se estimó en 70\% la probabilidad de transitar del primer grupo de edad al segundo.

Se asume que la mortalidad de residentes (R), así como de anestesistas activos de los dos tramos etarios $\left(\mathrm{E}_{1}\right.$ $\mathrm{y}_{2}$ ) es despreciable.
Para evaluar la bondad de ajuste de los resultados de modelo se compararon los datos empíricos históricos y los simulados. Se examinó la oferta de médicos simulada por el modelo y el número de médicos observados en el período 2000-2017, para el cual se contaba con datos históricos. Se calculó el error relativo (e) y la raíz cuadrada de la media de los cuadrados de los errores relativos (rRMSE) de la oferta de especialistas:

$$
\begin{gathered}
e_{t}=\frac{\left(Y_{t}-Y_{t}\right)}{Y_{t}} \\
\text { rRMSE }=\sqrt{\frac{\sum_{t=1}^{n} e_{t}^{2}}{n}}
\end{gathered}
$$


Donde $y_{t}$ representa el número observado de especialistas en cada año del período 2000-2017; $\hat{y}_{t}$ representa el número de especialistas simulado por el modelo de oferta.

Los años 2016 y 2017 fueron utilizados para valorar la calidad del modelo de forma independiente al ajuste de los parámetros realizado con los años previos. Los datos fueron obtenidos del Sistema de Control y análisis de recursos humanos del Ministerio de Salud Pública (SCARH).

Se estableció que el modelo era preciso, si el rRMSE estaba por debajo de o,1 siguiendo los valores propuestos por (1).

El año óptimo de inicio de la proyección de oferta se fijó como aquel que minimizó el rRMSE. Ante cambios en el año de inicio de la simulación, se consideró el año que hacía mínimo el rRMSE.

Para determinar el valor óptimo de estudiantes que ingresan por año a formarse en la especialidad, se consideró, entre el ingreso promedio y el valor máximo histórico, aquel valor que minimizó el rRMSE.

\section{Modelo de demanda/necesidad}

Para establecer la demanda/necesidad se consideró el total de horas de anestesistas (849 222 anuales) estimado para el año 2011 , en un estudio previo (4). A partir una carga horaria de trabajo de 48 horas por semana, durante 43 semanas laborales al año, se determinó una necesidad de profesionales de 411 .

Los datos sobre población fueron obtenidos de las proyecciones del INE para el período 2011-2050.

Se determinó la necesidad de especialistas por cada 100 ooo habitantes. Se proyectó la necesidad hacia 2050 teniendo en cuenta que la tasa de crecimiento de dicha necesidad sería de 1,3\% anual; es decir, una tasa acumulada de aumento de la necesidad de especialistas de $65,53 \%(26-27)$.
Como alternativa, se consideró un crecimiento moderado de $0,6 \%$ anual, que representa una tasa acumulada de aumento de la necesidad de 26,33\% (28).

Se proyectó la brecha absoluta y relativa. Se consideró el criterio de Tess y Amstrong (23, p86).

$$
\text { Brecha_Relativa }{ }_{t}=\frac{\text { Oferta }_{t}-\text { Necesidad }_{-}}{\text {Oferta }_{t}} * 100
$$

Este criterio establece una serie de rangos para definir:

- Déficit claro: déficit de $+10 \%$ o mayor

- Déficit leve: entre $-5 \%$ y $-10 \%$

- Equilibrio: entre $-5 \%$ y $+5 \%$

- $\quad$ Superávit leve: entre $+5 \%$ y $+10 \%$

- Superávit claro: $+10 \%$ o mayor

- La proyección de demanda/necesidad inicia en el año 2011; por tanto, la brecha se calcula para el período 2011-2050.

\section{RESULTADOS}

\section{Modelo de oferta}

El año 1997 es el que minimiza el rRMSE para el período considerado, el cual se toma como punto de partida para la proyección de la oferta.

En la proyección de oferta de especialistas hacia 2050, se observó que, si bien los primeros años son de crecimiento, luego se llega a una asíntota. El total de residentes rápidamente se nivela en un total de 90 estudiantes.

En la Figura 2 se observó la evolución del total de anestesistas proyectados por el modelo. Los puntos rojos representan los datos reales para los años 2000 a 2015 ; los puntos verdes en el gráfico corresponden al total de especialistas para los años 2016 y 2017 . Estos fueron incorporados a posteriori de ajustado el modelo.

Figura 2. Total de anestesistas, período 1997-2050

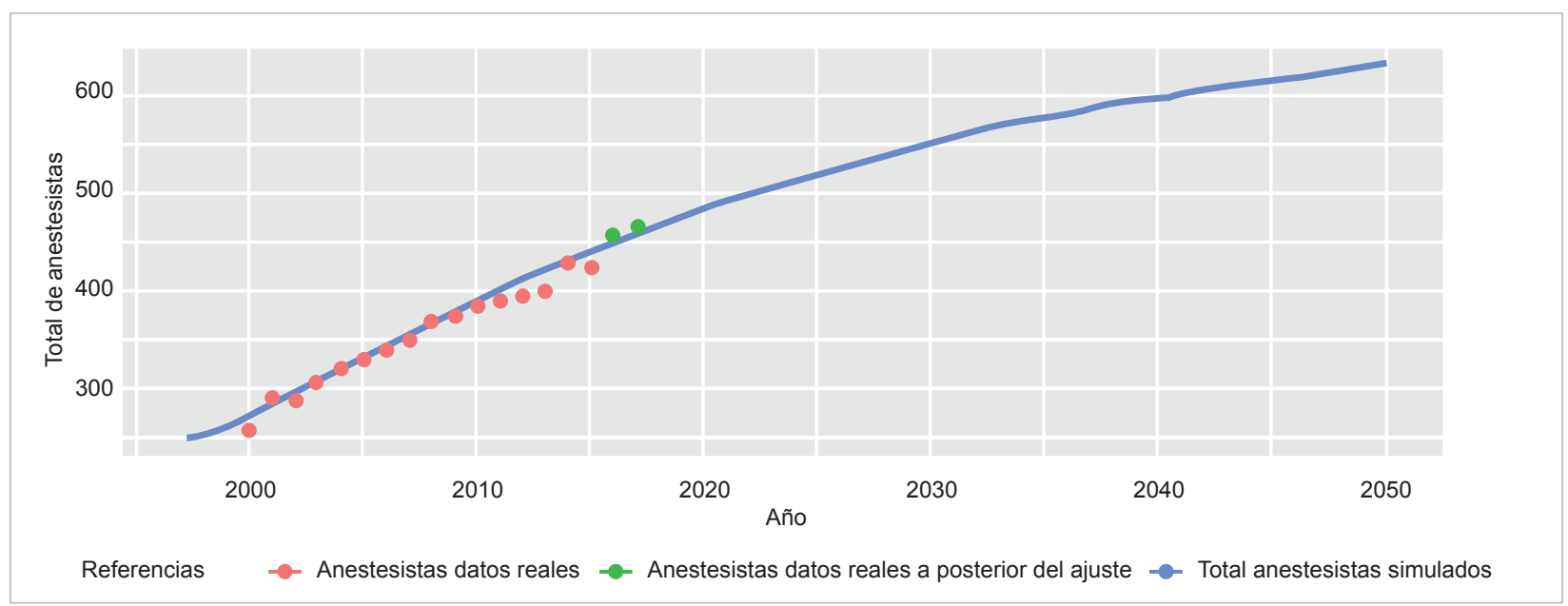


Se compararon datos históricos y simulados para evaluar el modelo (Tabla 1) y el rRMSE. El rRMSE fue o,0245, marcadamente inferior a 0,1 , por lo que se consideró que el modelo es apropiado para esta situación.

Tabla 1. Comparación de datos simulados por el modelo de la oferta de anestesistas con reales

\begin{tabular}{|c|c|c|c|c|c|c|c|c|c|c|c|c|c|c|c|c|c|c|c|}
\hline Año & 2000 & 2001 & 2002 & 2003 & 2004 & 2005 & 2006 & 2007 & 2008 & 2009 & 2010 & 2011 & 2012 & 2013 & 2014 & 2015 & 2016 & 2017 & rRMSE \\
\hline $\begin{array}{c}\text { Datos } \\
\text { simulados }\end{array}$ & 271 & 282 & 293 & 304 & 316 & 328 & 340 & 351 & 363 & 375 & 386 & 397 & 407 & 417 & 427 & 436 & 445 & 454 & 0,0245 \\
\hline $\begin{array}{l}\text { Datos } \\
\text { históricos }\end{array}$ & 260 & 288 & 290 & 306 & 319 & 328 & 337 & 348 & 366 & 373 & 380 & 387 & 391 & 395 & 425 & 422 & 452 & 459 & \\
\hline$e^{2}$ & 0,002 & 0,000 & 0,000 & 0,000 & 0,000 & 0,000 & 0,000 & 0,000 & 0,000 & 0,000 & 0,000 & 0,001 & 0,002 & 0,003 & 0,000 & 0,001 & 0,000 & 0,000 & \\
\hline
\end{tabular}

Se analizó el efecto en el rRMSE al variar el cupo anual de la residencia de 15 a 30 personas (Figura 3).

Los cupos de ingreso a la especialidad que presentan elmínimo de rRMSE son 22 y 23 estudiantes por año, con un rRMSE de 0,023 y 0,0245 respectivamente. $\mathrm{Cu}$ pos de 18 y 27 representan los límites a partir de los cuales el rRMSE supera 0,1 .

Figura 3. rRMSE de la oferta de especialistas por variación en los cupos de ingreso a la especialidad

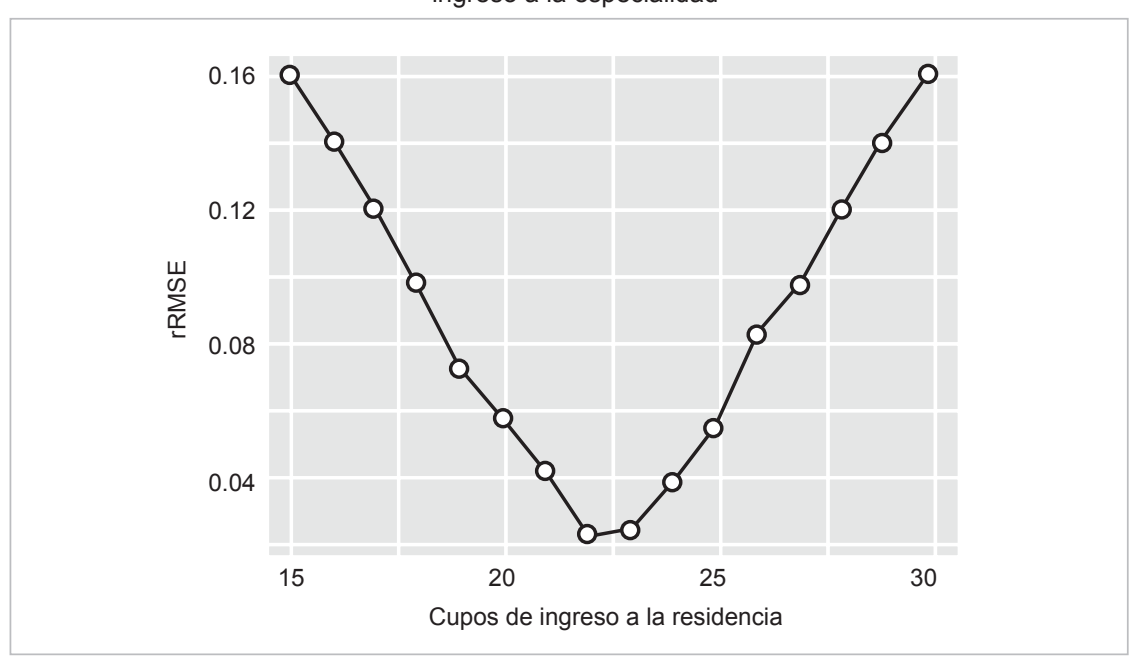

En la Tabla 2 se presentan las proyecciones de los modelos de oferta, denecesidad y la brecha relativa. Para el período considerado, la situación es de equilibrio hasta el año 2033; luego, entre 2035 y 2040, habría un déficit leve, y desde el 2041 a 2050, un déficit claro.

$\mathrm{Al}$ final del período de proyección, con un crecimiento acumulado de $65,5 \%$, la necesidad pasa a ser de 19,9 cada 100 ooo habitantes, es decir, 738 especialistas.

Si no se hubiese considerado el crecimiento poblacional (población constante en el período de 3412 636) la necesidad hubiese evolucionado de 411 en 2011 (12,0 cada 100 ooo habitantes) a 680 en 2050 (19,9 cada 100 ooo habitantes).

Cuando se considera una tasa de crecimiento de la necesidad de $0,6 \%$ anual, se llega a 15,2 por cada
100 ooo habitantes en 2050. Si se mantiene el resto de los parámetros del modelo constantes respecto al escenario anterior, se proyecta una situación de equilibrio hasta 2018 y luego de superávit leve.

Si el cupo de ingresos en la especialidad fuese de 20 por año, lo que implicaría un total de 80 estudiantes anuales, se seguiría teniendo un modelo de oferta con un rRMSE de 0,057 dentro de lo esperado. Si además se considerara un crecimiento moderado de la necesidad de $0,6 \%$ anual, se tendría para el año 2011 una situación de déficit claro; entre 2012 y 2017, déficit leve, y luego un equilibrio hasta 2050. En cambio, si el crecimiento fuese de 1,3\% anual se tendría una situación de déficit claro en todo el período. 
Tabla 2. Proyecciones de oferta, demanda/necesidad y brecha relativa porcentual 2011-2050

\begin{tabular}{|c|c|c|c|c|c|c|}
\hline Año & $\begin{array}{c}\text { Proyección de } \\
\text { población }\end{array}$ & $\begin{array}{c}\text { Tasa de necesidad } \\
\text { por } 100000 \\
\text { habitantes }\end{array}$ & $\begin{array}{l}\text { Necesidad de } \\
\text { anestesistas }\end{array}$ & $\begin{array}{c}\text { Oferta de } \\
\text { anestesistas }\end{array}$ & $\begin{array}{c}\text { Brecha } \\
\text { relativa (\%) }\end{array}$ & $\begin{array}{c}\text { Categoría de } \\
\text { brecha }\end{array}$ \\
\hline 2011 & 3412636 & 12,04 & 411 & 397 & $-3,5$ & Equilibrio \\
\hline 2012 & 3426466 & 12,20 & 418 & 407 & $-2,7$ & Equilibrio \\
\hline 2013 & 3440157 & 12,36 & 425 & 417 & $-2,0$ & Equilibrio \\
\hline 2014 & 3453691 & 12,52 & 432 & 427 & $-1,3$ & Equilibrio \\
\hline 2015 & 3467054 & 12,68 & 440 & 436 & $-0,8$ & Equilibrio \\
\hline 2016 & 3480222 & 12,85 & 447 & 445 & $-0,5$ & Equilibrio \\
\hline 2017 & 3493205 & 13,01 & 454 & 454 & $-0,1$ & Equilibrio \\
\hline 2018 & 3505985 & 13,18 & 462 & 462 & 0,0 & Equilibrio \\
\hline 2019 & 3518552 & 13,35 & 470 & 470 & 0,1 & Equilibrio \\
\hline 2020 & 3530912 & 13,53 & 478 & 478 & 0,1 & Equilibrio \\
\hline 2021 & 3543026 & 13,70 & 485 & 486 & 0,1 & Equilibrio \\
\hline 2022 & 3554915 & 13,88 & 493 & 493 & $-0,1$ & Equilibrio \\
\hline 2023 & 3566550 & 14,06 & 501 & 500 & $-0,3$ & Equilibrio \\
\hline 2024 & 3577896 & 14,25 & 510 & 507 & $-0,6$ & Equilibrio \\
\hline 2025 & 3588937 & 14,43 & 518 & 514 & $-0,8$ & Equilibrio \\
\hline 2026 & 3599663 & 14,62 & 526 & 520 & $-1,2$ & Equilibrio \\
\hline 2027 & 3610035 & 14,81 & 535 & 526 & $-1,6$ & Equilibrio \\
\hline 2028 & 3620004 & 15,00 & 543 & 532 & $-2,1$ & Equilibrio \\
\hline 2029 & 3629541 & 15,20 & 552 & 538 & $-2,5$ & Equilibrio \\
\hline 2030 & 3638632 & 15,39 & 560 & 544 & $-2,9$ & Equilibrio \\
\hline 2031 & 3647229 & 15,59 & 569 & 549 & $-3,6$ & Equilibrio \\
\hline 2032 & 3655289 & 15,80 & 578 & 554 & $-4,2$ & Equilibrio \\
\hline 2033 & 3662795 & 16,00 & 586 & 559 & $-4,8$ & Equilibrio \\
\hline 2034 & 3669744 & 16,21 & 595 & 564 & $-5,5$ & Déficit leve \\
\hline 2035 & 3676092 & 16,42 & 604 & 569 & $-6,1$ & Déficit leve \\
\hline 2036 & 3681848 & 16,63 & 612 & 573 & $-6,9$ & Déficit leve \\
\hline 2037 & 3687000 & 16,85 & 621 & 577 & $-7,7$ & Déficit leve \\
\hline 2038 & 3691553 & 17,07 & 630 & 581 & $-8,5$ & Déficit leve \\
\hline 2039 & 3695520 & 17,29 & 639 & 585 & $-9,2$ & Déficit leve \\
\hline 2040 & 3698924 & 17,52 & 648 & 589 & $-10,0$ & Déficit leve \\
\hline 2041 & 3701762 & 17,74 & 657 & 593 & $-10,7$ & Déficit claro \\
\hline 2042 & 3704055 & 17,97 & 666 & 597 & $-11,5$ & Déficit claro \\
\hline 2043 & 3705850 & 18,21 & 675 & 600 & $-12,5$ & Déficit claro \\
\hline 2044 & 3707145 & 18,44 & 684 & 603 & $-13,4$ & Déficit claro \\
\hline 2045 & 3707919 & 18,68 & 693 & 606 & $-14,3$ & Déficit claro \\
\hline 2046 & 3708236 & 18,93 & 702 & 609 & $-15,3$ & Déficit claro \\
\hline 2047 & 3708094 & 19,17 & 711 & 612 & $-16,2$ & Déficit claro \\
\hline 2048 & 3707490 & 19,42 & 720 & 615 & $-17,1$ & Déficit claro \\
\hline 2049 & 3706463 & 19,67 & 729 & 618 & $-18,0$ & Déficit claro \\
\hline 2050 & 3705000 & 19,93 & 738 & 621 & $-18,9$ & Déficit claro \\
\hline
\end{tabular}

\section{DISCUSIÓN}

Se propone un modelo para proyectar los desequilibrios entre la oferta y demanda/necesidad de anestesistas en Uruguay, el cual fue diseñado en un programa de libre acceso que reproduce satisfactoriamente la dinámica de la realidad. Se caracteriza por ser un modelo simple que utiliza información disponible en los países (Figura 1) y un programa sin costo adicional. A la vez, presenta la bondad de que es posible complejizarlo en sucesivas etapas, incorporando nuevas variables y nuevos vínculos entre las mismas, a fin de mejorar las proyecciones realizadas (18).

Asimismo, podría adaptarse a nuevas perspectivas de planificación, basada en equipos de salud y no solo enespecialidades, contemplar los movimientos migratorios crecientes que afectan tanto la oferta de recursos humanos como la demanda/necesidad, variabilidad por áreas geográficas, tiempo de respuesta a la atención médica, y/o estudiar servicios específicos (públicos o privados). 
Cabe destacar que la proyección de brecha para el caso de Uruguay es de equilibrio en la mayor parte del período analizado, siendo hasta el 2033 una brecha inferior al 5\%, constándose un problema de déficit claro a partir del 2040. Estas cifras se sostienen siempre que se cumplan los criterios definidos y las condiciones de oferta y demanda/necesidad (Tabla 2 ).

Se trata de una proyección desde la perspectiva del sistema de salud en su conjunto. Por ello no se descarta que puedan existir desequilibrios por región. Dada la situación del mercado de trabajo en el país, una condición necesaria para definir el equilibrio es que los anestesistas se desempeñen de acuerdo con la forma de trabajo con la cual se definió la necesidad (cargos de 48 horas semanales).

Se utilizó una tasa de crecimiento anual de necesidad, de $1,3 \%$, a partir de las recomendaciones de estudios similares. En el otro extremo, si se considerara una tendencia estable de necesidad de o,6\% anual y el resto de las variables sin modificar, se proyecta un superávit leve a partir del 2018 y hasta el final del período de proyección.

Otro elemento por destacar del modelo es que incorpora información histórica para definir parámetros del modelo y evaluar la validez de las proyecciones.

Los resultados de la Tabla 1 muestran un valor de rRSME claramente inferior a 0,1 , umbral de referencia $(1,10)$. Este último componente se considera relevante, dado que en una reciente revisión se constata que la mayoría de los modelos examinados carecen de indicadores de validación (5).

También permite realizar el análisis de escenarios al variar el cupo de ingresos a la especialidad, ya sea por incremento de las plazas de formación y/o por solicitud de revalidación de títulos desde el exterior que aportan nuevos insumos para quienes toman decisiones

Conflictos de intereses: Ninguno.

\section{REFERENCIAS}

1. Ishikawa T, Fujiwara K, Ohba H, Suzuki T, Ogasawara K. Forcasting the regional distribution and sufficiency of physicians in Japan with a coupled system dynamics-geographic information system model. Hum Resour Health. 2017; 15(1):64. https://doi.org/10.1186/s12960-017-0238-8.

2. World Health Organization. Global strategy for Human resources for health: Workforce 2030 [Internet]. Geneva: WHO; 2016 [cited 2021 Feb 3]. https://bit.ly/3locbqV.

3. Ministerio de Salud de la República Oriental del Uruguay. Jornadas de Intercambio. A 10 años de iniciada la reforma sanitaria: evaluación y desafíos del Sistema Nacional Integrado de Salud [Internet]. Montevideo: Ministerio de Salud, OMS, OPS; 2016 [cited 2021 Fe 3]. https://bit.ly/3lp3dd7.

4. Ley 19301 de 2014. Poder Ejecutivo de la República Oriental del Uruguay. Sistema Nacional de Residencias Médicas [Internet]. 2014 [cited 2021 Mar 2]. https://bit.ly/31mfhEz.

5. Safarishahrbiri A. Workforce forecasting models: A systematic review. Journal of Forecasting. 2018;1-15.
6. Roberfroid D, Leonard C, Stordeur S. Physician supply forecast: better than peering in a crystal ball? Human Resources for Health. 2009; 7:10. https://doi.org/10.1186/1478-4491-7-10.

7. Homer JB, Hirsch GB. System Dynamics Modeling for Public Health: Background and Opportunities. AJPH. 2006; 96:452-8. https://doi. org/10.2105/AJPH.2005.062059.

8. AvokaAsamani J, Chebere M, Barton P, D'Almeida S, Ankrah E, Oppong R. Forecast of Healthcare Facilities and Health Workforce Requirements for the Public Sector in Ghana, 2016-26. Int Health Policy Manag. 2018; 1-13. https://doi.org/10.15171/ijhpm.2018.64.

9. Laurence C, Karnon J. Improving the planning of the GP workforce in Australia: a simulation model incorporating work transitions, health need and service usage. Human Resources for Health. 2016; 14(13). https://doi.org/10.1186/s12960-016-0110-2.

10. Ishikawa T, Ohba H, Yokooka Y, Nakamura K, Ogasawara K. (2013). Forecasting the absolute and relative shortage of physicians in Japan using a system dynamics model approach. Human Resources for Health. 2013; 11:41. https://doi.org/10.1186/1478-4491-11-41.

11. Xu Z, Coors V. Combining system dynamics model, GIS and 3D visualization in sustainability assessment of urban residential development. Building and Environment. 2012; 47:272-87. https://doi.org/10.1016/j. buildenv.2011.07.012.

12. Guan D, Gao W, Su W, Li H, Hokao K. Modeling and dynamic assessment of urban economy-resource-environment system with a coupled system dynamics - geographic information system model. Ecological Indicators. 2011; 11(5):1333-44. https://doi.org/10.1016/j. ecolind.2011.02.007.

13. Senese F, Tubertini P, Mazzocchetti A, Lodi A, Rouzi C, Grilli R. Forecasting future needs and optimal allocation of medical residency positions: the Emilia-Romagna Region case study. 2015; 13(1):7. https://doi.org/10.1186/1478-4491-13-7.

14. Ansah J, de Korne D, Bayer S, Pan C, Jayabaskar T, Matchar D, Lew N, Phua A, Koh V, Lamoreaux E, Quek D. Future requirements for and supply of ophthalmologists for an aging population in Singapore. 2015; 13:86. https://doi.org/10.1186/s12960-015-0085-4.

15. CFWI. Future demand for skills: Initial Result. Horizon 2035. Health and care workforce futures [Internet]. United Kingdom; 2015 [cited 2021 Feb 3]. https://bit.ly/3xQHSOz.

16. CFWI. In-depth review of the general practitioner workforce. Final report [Internet]. United Kingdom; 2014 [cited 2021 Feb 3]. https://bit.ly/3IrRs5q.

17. CFWI. In-depth review ot the anaesthetics and intensive care medicine workforce. Final report [Internet]. United Kingdom; 2015 [cited 2021 Feb 3]. https://bit.ly/3pffpoU.

18. Cave S, Willis G, Woodward A. A restrospective of System Dynamics based workforce modelling at the Centre for Workforce Intelligece. 2016 [cited 2021 Mar 6]. https://bit.ly/3pkVcqP.

19. Masnick K, McDonnell G. A model linking clinical workforce skill mix planning to health and health care dynamics. Human Resources for Health. 2010; 8:11. https://doi.org/10.1186/1478-4491-8-11.

20. Barber P, López-Valcárcel BG. Forecasting the need for medical specialists in Spain: application of a system dynamics model. Hum ResourHealth. 2010; 8:24. https://doi.org/10.1186/1478-4491-8-24.

21. Barber P. Necesidad de recursos humanos de alto nivel en el sector sanitario, Panamá 2014-2025 [Internet]. Gran Canaria: Equipo de Economía de la Salud de la Universidad de Las Palmas de Gran Canaria; 2015 [citado 2021 Mar 4]. https://bit.ly/3pklJ7A.

22. Barber P, Gonzales López-Valcárcel B, Suárez R. Oferta, demanda y necesidad de médicos especialistas en Brasil. Proyecciones a 2020 [Internet]. Sociedade Brasileira de Medicina de Família e Comunidade (SBMFC); 2012. https://bit.ly/3xJlqG4.

23. Barber Pérez P, González López-Valcárcel B. Oferta y necesidad de médicos especialistas en España (2006-2030) [Internet]. Gran Canaria: Universidad de Las Palmas de Gran Canaria; 2007 [cited 2021 Mar 6]. https://bit.ly/3G5gaRd. 
24. Barber Pérez P, González López-Valcárcel B. Estimación de Oferta y demanda de médicos especialistas. España (2018-2030) [Internet]. Gran Canaria: Universidad de Las Palmas de Gran Canaria; 2018 [cited 2021 Mar 6]. https://bit.ly/2ZLFxl7.

25. Ministerio de Salud Pública. Sistema Nacional Integrado de Salud. División RRHH. Estimación de las necesidades de anestesistas [Internet]. Montevideo: MSP; 2012 [cited 2021 Mar 6]. https://bit.ly/2ZO0pOZ

26. Cavalleri F, Castromán P, Rodríguez A, Riva J. Utilidad de un método de simulación para evaluar la oferta y demanda de anestesiólogos en Uruguay (2011-2025): propuesta para prospección y planificación de necesidad de recursos humanos en Anestesiología. Rev Méd Urug [Internet]. 2013 [cited 2021 Mar 6]; 29(3):165-73. https://bit.ly/3lqBZCD.

27. Egger Halbeis $C B$, Schubert A. Staffing the operating room suite: perspectives from Europe and North America on the role of different anesthesia personnel. Anesthesiol Clin.2008; 26(4):637-63. https:// doi.org/10.1016/j.anclin.2008.07.002.

28. Physician Supply and Demand: Projections to 2020 [Internet]. U.S. Department of Health and Human Services Health Resources and Services Administration. Bureau of Health Proffesions.2006 [cited 2021 Mar 6]. https://bit.ly/32JUYSg. 